\title{
Efficacy of Monitoring for Multiple Original Antiplatelet Therapy during Intracranial Stent Placement: A Preliminary Study
}

Yasuo Suga, ${ }^{1}$ Hidenori Oishi, ${ }^{1,2}$ Munetaka Yamamoto, ${ }^{1}$ and Hajime Arai ${ }^{1}$

Objective: During cerebral aneurysm embolization using intracranial stents, platelet aggregation increases owing to increased wall shear stress and a loss of vascular endothelial function at the stent implantation site. Preoperative multiple antiplatelet therapy was introduced to prevent severe thromboembolic complications due to increased platelet aggregation. However, specific guidelines for the administration and pharmacological evaluation of this therapy do not exist currently. We examined the benefits of perioperative platelet aggregation monitoring in a cohort of patients.

Methods: We had 377 patients with unruptured intracranial aneurysms who underwent stent-assisted embolization at our hospital between December 2012 and November 2019. We ultimately included 181 patients in our final analysis. These patients were continuously administered aspirin (100 mg/day) and clopidogrel $(75 \mathrm{mg} /$ day) for more than 5 days before the procedure to the post-procedural period. Of these patients, 30 patients who underwent light transmission aggregometry (LTA) before procedure, post-procedure ( 3 days after procedure), and at first post-discharge clinic visit were included as the subjects. The following characteristics were studied: age; sex; presence/absence of hypertension, dyslipidemia, and/or diabetes mellitus; location of aneurysm; type/number of stent; technique for stent placement; duration of preoperative multiple antiplatelet therapy; perioperative platelet aggregation test results; and postoperative ischemic or hemorrhagic complications. Results: Among these 30 patients, the median duration of antiplatelet therapy prior to the preoperative platelet aggregation measurements was 7 (interquartile range [IQR]: 6-8) days, and post-discharge measurement of LTA was performed at a median period of 27 (IQR: 22-35.5) days after procedure. The preoperative, postoperative, and first post-discharge clinic visit LTA values for adenosine diphosphate (ADP)-induced platelet aggregation were 50\% (IQR: 44-54\%), 42.5\% (IQR: $36-48 \%$ ), and 36\% (IQR: 32-40\%), respectively. These results represented gradual decrease in LTA values and a significant difference between the preoperative and post-discharge values. The LTA values for collagen aggregation showed a significant difference evident between the preoperative and post-discharge values; preoperative 38\% (IQR: 27-60\%), postoperative $42 \%$ (IQR: 30-58\%), post-discharge $28 \%$ (IQR: 20-42\%), respectively. We had one thromboembolic complication and one hemorrhagic complication. The results indicated that appropriate platelet aggregation monitoring during multiple antiplatelet therapy prevents thromboembolic complications such as stent thrombosis. However, we also found that many patients demonstrated increased postoperative platelet aggregation inhibitory effects due to the postoperative continuation of the same multiple antiplatelet therapy that was used preoperatively.

Conclusion: This study demonstrates that postoperative, continuous, oral antiplatelet therapy induces increased platelet aggregation inhibition effects, which may lead to hemorrhagic complications. Therefore, continued platelet aggregation monitoring after surgery may be important to allow for any necessary alterations to the therapeutic dose and regimen.

Keywords $>$ antiplatelet therapy, intracranial aneurysm, monitoring, platelet aggregation test, stent

${ }^{1}$ Department of Neurosurgery, Juntendo University School of Medicine, Tokyo, Japan

${ }^{2}$ Department of Neuroendovascular Therapy, Juntendo University

School of Medicine, Tokyo, Japan

Received: June 26, 2020; Accepted: November 9, 2020

Correspondence author: Yasuo Suga. Department of Neurosurgery, Juntendo University School of Medicine, 2-1-1, Hongo, Bunkyo-ku, Tokyo 113-8421, Japan

Email: yssuga@juntendo.ac.jp

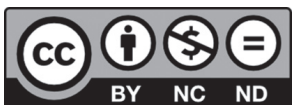

This work is licensed under a Creative Commons Attribution-NonCommercialNoDerivatives International License. 


\section{Introduction}

The usefulness of perioperative antiplatelet therapy for the prevention of thromboembolic complications arising from coil embolization of unruptured intracranial aneurysms has been established. ${ }^{1,2)}$ However, during stent-assisted coil embolization and flow-diverter placement for intracranial aneurysms, the elevation in wall shear stress at the stent implantation site may lead to impaired vascular endothelial function, increased platelet aggregation, and increased perioperative thromboembolic complications. ${ }^{3-5}$ Because these complications may become severe, prophylactic multiple antiplatelet therapy is typically introduced preoperatively. ${ }^{6-8)}$ Platelet aggregation measurements prior to intracranial stent placement are now frequently performed to evaluate multiple antiplatelet therapy. However, a consensus on the dosing regimen and drug efficiency monitoring has not yet been reached. While consensus regarding percutaneous coronary stent placement has been reached in the field of cardiovascular medicine, ${ }^{9,10)}$ applying the same criteria to cerebral endovascular treatment may not be appropriate, ${ }^{3,11,12)}$ Therefore, developing criteria for cerebral endovascular treatment is critical.

Although a large-scale, prospective study may be the most effective way to develop the criteria for cerebral endovascular treatments, insufficient inhibition of platelet aggregation may lead to severe thromboembolic complications. Therefore, conducting such a study in routine clinical settings is difficult. Hence, in many cases, dose and therapeutic regimens must be adjusted based on the results of platelet aggregation tests. In addition, clear evidence is not available regarding the interpretation of the results and subsequent dose adjustments, making it difficult to reach a consensus. Although preoperative platelet aggregability is often discussed, postoperative platelet aggregability monitoring is rarely reported; dose reduction or discontinuation of the multiple antiplatelet therapy may be an important issue.

Therefore, we examined the benefits of drug efficacy monitoring by continuously measuring and analyzing platelet aggregability in patients who were candidates for stent-assisted embolization of intracranial aneurysms at our institution.

\section{Materials and Methods}

\section{Patients}

The study participants were allowed to opt-out, and the study design was approved by the Ethics Committee of

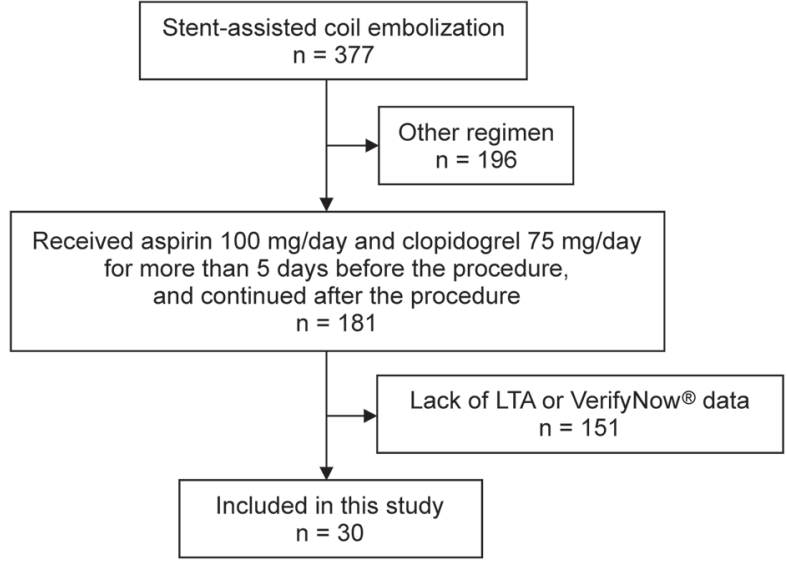

Fig. 1 Flow diagram of patient selection

Juntendo University Hospital (Approval No. 19-203). A total of 377 patients who underwent stent-assisted coil embolization for intracranial aneurysms between December 2012 and November 2019 and received preoperative and perioperative multiple antiplatelet therapy consisting of aspirin, clopidogrel, and cilostazol, and were included in this retrospective analysis. Among these, 196 patients were excluded because of the variance in antiplatelet therapy regimen. The remaining 181 patients who received aspirin at $100 \mathrm{mg} /$ day and clopidogrel at $75 \mathrm{mg} /$ day for more than 5 days prior to the preoperative platelet aggregation measurements and continued the same antiplatelet therapy after procedure, since clopidogrel takes 3-5 days for its platelet aggregation inhibitory effects to reach steady state, were included in the study. Of these 181 patients, 30 patients were finally included as the subjects, who underwent the light transmission aggregometry (LTA) and VerifyNow before procedure as well as underwent LTA 3 days after procedure and after discharge at outpatient setting. The remaining 151 patients were excluded from the study (Fig. 1). The following characteristics were studied: age; sex; presence/absence of hypertension, dyslipidemia, and/ or diabetes mellitus; location of aneurysm; type/number of stent; technique for stent placement; duration of preoperative multiple antiplatelet therapy; perioperative platelet aggregation test results; and postoperative ischemic or hemorrhagic complications.

\section{Platelet aggregation test}

Platelet aggregability was evaluated using both LTA (MCM HEMA TRACER 313M; MC MEDICAL, Tokyo, Japan) and VerifyNow (Accumetrics, San Diego, CA, USA). Although LTA is the current gold standard for platelet aggregation testing, VerifyNow is a point-of-care testing 


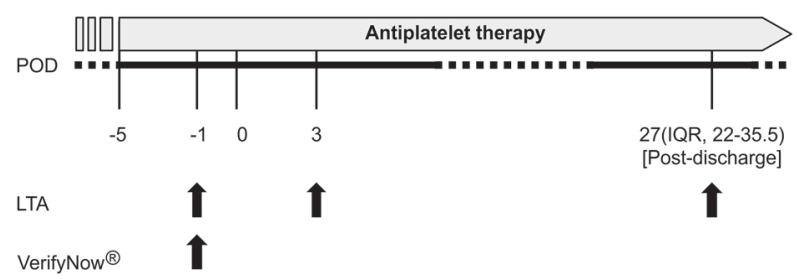

Fig. 2 Regimen for platelet aggregation test. IQR: interquartile range; POD: postoperative day

device that demonstrates a high correlation with LTA. ${ }^{13-16)}$ Although both tests measure light transmission through samples to detect changes caused by platelet aggregation, LTA uses plasma samples, whereas the VerifyNow instrument uses whole blood samples. Because LTA is an expensive and cumbersome procedure, many studies use point-of-care testing devices, such as VerifyNow, as an alternative. However, LTA is approved in Japan for the on-label indication of platelet aggregation testing, and is thus covered by national health insurance, whereas VerifyNow is not. Therefore, LTA was used for both preoperative and postoperative platelet aggregation testing, whereas VerifyNow was used only for preoperative testing. Thus, we mainly used LTA for the evaluation of platelet aggregation, and VerifyNow was used accessorily when we evaluated preoperative platelet aggregation. As shown in Fig. 2, preoperative testing using LTA and VerifyNow occurred 1 day before surgery, except for 1 case which was tested 3 days before procedure. Postoperative platelet aggregation testing 3 days after surgery and at first visit outpatient after discharge was performed with only LTA.

For LTA, the effective cutoff values for ADP-induced platelet aggregation and collagen aggregation were set at $\leq 50 \%$ and $\leq 60 \%$, respectively. For VerifyNow testing, the effective cutoff values for the P2Y12 reaction unit (PRU) and aspirin reaction unit (ARU) were set at $\leq 230$ and $\leq 550$, respectively. The cutoff scores were based on previously published results. ${ }^{7,10,17,18)}$ Despite significant correlation between LTA and VerifyNow results, ${ }^{13)}$ they are not always considered equally effective for determining platelet aggregation. Hence, we considered patients with results above the cutoff value in both tests to be resistant to the antiplatelet therapy, whereas other patients were considered not to be resistant. The patients were divided into the suspected clopidogrel-resistance group (i.e., patients with both ADP-induced platelet aggregation and PRU values above the cutoff value) and the suspected aspirin-resistance group (i.e., patients with both collagen-induced platelet aggregation and ARU values above the cutoff value).

\section{Endovascular procedures}

We performed all procedures under general anesthesia and inserted $6 \mathrm{~F}$ guiding sheath via right common femoral artery, and catheterized $6 \mathrm{~F}$ distal access catheter to intracranial internal carotid or vertebral artery. In most cases, we placed the stent before coil insertion into the aneurysm using the jailing technique, and in some cases we used the stent as a rescue stent after coil embolization. Stent-assisted coil embolization may involve complications related to stent placement, including thromboembolism and incomplete stent apposition. Therefore, extra caution was taken to avoid stent fracture and incomplete stent apposition to the parent vessel, and if necessary, adequate stent expansion and stent apposition to the vascular wall was performed using a balloon catheter. Intraoperatively, heparin (4000-6000 U) was administered intravenously after sheath insertion, with additional doses provided to maintain an activated clotting time $>250 \mathrm{~s}$. After the procedure, we did not add any antithrombotic drugs to aspirin at $100 \mathrm{mg}$ /day and clopidogrel at $75 \mathrm{mg} /$ day in most cases. In some cases, we used ozagrel sodium at $160 \mathrm{mg} /$ day for 3-7 days or argatroban hydrate at 30-60 mg/day for 2-3 days.

\section{Statistical analysis}

Continuous variables were reported as median interquartile range (IQR) and categorical variables were reported as counts and percentage. The Friedman test was performed to test for significant changes of LTA at three different timings (preoperative, postoperative at day 3 , and post-discharge). The Wilcoxon signed-rank tests were performed to compare the differences between two measurements. A significance threshold of 0.01 was chosen for statistical analysis. This is slightly more conservative than Bonferroni's correction, with a threshold of 0.01 that accounts for the three different timings analyzed $(0.05 / 3=0.017)$.

\section{Results}

The characteristics of the patients are shown in Table 1. Of the 30 patients, 7 were males and 23 were females, with a median age of 67 (IQR: 54-72) years. Past medical history included hypertension in 19 patients, dyslipidemia in 7 patients, and diabetes in 3 patients. The location of aneurysms of these patients is shown in Table 2. The median duration of antiplatelet therapy prior to the preoperative platelet aggregation measurements was 7 (IQR: 6-8) days. Post-discharge measurement of LTA was performed at a median period of 27 (IQR: 22-35.5) days after procedure. 
Suga $Y$, et al.

Table 1 Patient background

\begin{tabular}{lc} 
& Patients $(\mathrm{n}=30)$ \\
Median age & 67 years (IQR: $54-72)$ \\
Sex & Male $7(23 \%)$, Female 23 $(77 \%)$ \\
Hypertension & $19(63 \%)$ \\
Dyslipidemia & $7(23 \%)$ \\
Diabetes & $3(10 \%)$ \\
\hline
\end{tabular}

IQR: interquartile range

\begin{tabular}{lc} 
Table 2 Location of aneurysm (\%) \\
\hline ICA paraclinoid & $7(23)$ \\
VA & $6(20)$ \\
BA tip & $5(17)$ \\
ICA-PCoA & $5(17)$ \\
MCA & $3(10)$ \\
SCA & $2(7)$ \\
ACoA & $1(3)$ \\
PCA & $1(3)$
\end{tabular}

ACOA: anterior communicating artery; BA: basilar artery; ICA: internal carotid artery; MCA: middle cerebral artery; PCA: posterior cerebral artery; PCoA: posterior communicating artery; SCA: superior cerebellar artery; VA: vertebral artery
The Friedman test was conducted on 30 individuals to examine the effect at three different timings. ADP-induced platelet aggregation was found to be significantly different between the three timings $(\mathrm{p}<0.001)$. The preoperative, postoperative, and first post-discharge clinic visit LTA values for ADP-induced platelet aggregation were $50 \%$ (IQR: 44-54\%), 42.5\% (IQR: $36-48 \%$ ), and 36\% (IQR: $32-40 \%$ ), respectively. These results represented a statistically significant, but gradual decrease in LTA values and a significant difference between the preoperative and post-discharge values (Fig. 3). Collagen-induced platelet aggregation was also significantly different between the timings ( $\mathrm{p}<0.001)$. The LTA values for collagen aggregation showed a significant difference evident between the preoperative and post-discharge values with preoperative 38\% (IQR: 27-60\%), postoperative $42 \%$ (IQR: $30-58 \%$ ), and post-discharge 28\% (IQR: 20-42\%), respectively (Fig. 4). In comparison, the preoperative and post-discharge values in 25 of 30 patients had decreased in ADP-induced platelet aggregation, and 24 of 30 had decreased in collageninduced platelet aggregation. In VerifyNow, which was another modality that we used for the evaluation of preoperative platelet aggregation, the PRU and ARU values were 175 (IQR: 150-230) and 403 (IQR: 391-472), respectively. There were three patients in the suspected clopidogrel-resistant group, and there was none in the suspected aspirin-resistant group.

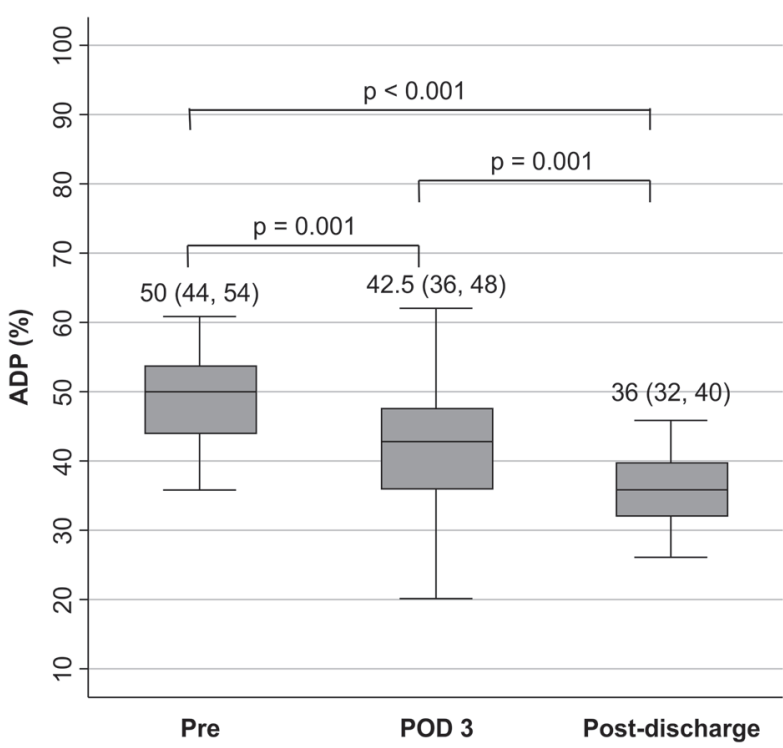

Fig. 3 Long-term changes in light transmission aggregometry (ADP), each number above the bar means median and interquartile range. Post-discharge measurement of LTA was performed at a median period of 27 (IQR: 22-35.5) days after procedure. ADP: adenosine diphosphate; LTA: light transmission aggregometry; POD: postoperative day; Pre: preoperative

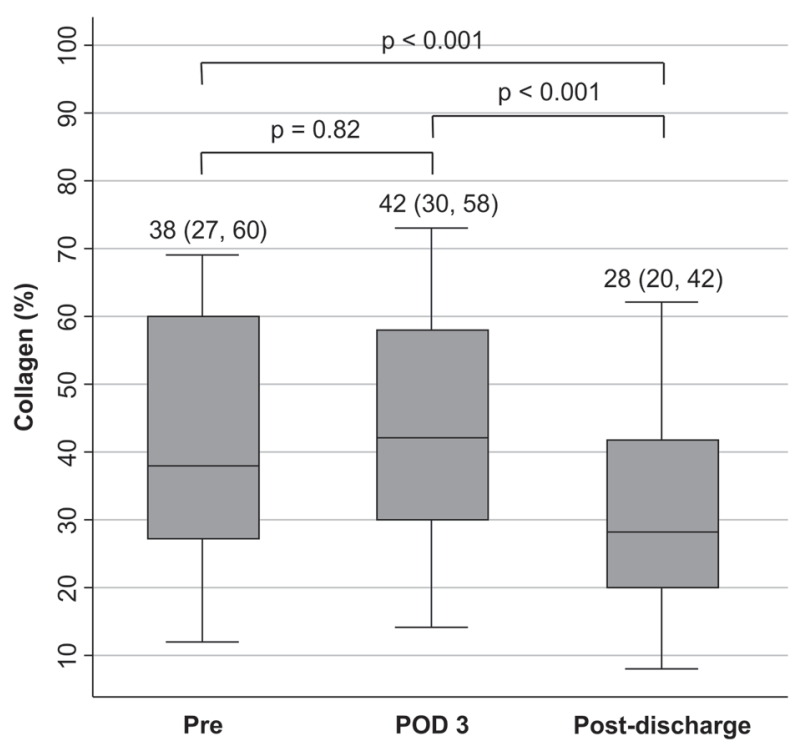

Fig. 4 Long-term changes in LTA (collagen), each number above the bar means median and interquartile range. Post-discharge measurement of LTA was performed at a median period of 27 (IQR: 22-35.5) days after procedure. LTA: light transmission aggregometry; POD: postoperative day; Pre: preoperative

No significant association was evident between the low response to clopidogrel and underlying diseases (hypertension, dyslipidemia, and diabetes).

Table 3 shows the type of implanted stents and technique of stent placement. In all, 25 patients had a single stent implanted, and the remaining 5 patients had dual 
Table 3 Type, number, and technique of stent placement

\begin{tabular}{lcccccc}
\multirow{2}{*}{ Type } & Cases & \multicolumn{5}{c}{ Technique } \\
\cline { 3 - 7 } & & Jail & Trans-cell & Combined $^{*}$ & Rescue & Others (dual stents) \\
Enterprise & 14 & 7 & 0 & 1 & 3 & 3 (overlap) \\
Neuroform EZ & 7 & 1 & 0 & 0 & 6 & 0 \\
LVIS Jr & 6 & 1 & 2 & 0 & 1 & 2 (Y-configuration) \\
Neuroform Atlas & 2 & 2 & 0 & 0 & 0 & 0 \\
LVIS Blue & 1 & 1 & 0 & 0 & 0 & 0 \\
Total number & 30 & 12 & 2 & 1 & 10 & 5 \\
\hline
\end{tabular}

${ }^{*}$ Combined means jail and trans-cell technique

stents implanted with overlapping technique or Y-configuration technique.

The complications observed in 3 of the 30 patients who underwent stent treatment included stent thrombosis in one patient, subarachnoid hemorrhage in one patient due to pullout injury of small branch of middle cerebral artery, and subcutaneous hematoma in one patient at outpatient visit. The patient who had stent thrombosis experienced transient left hemiparesis, conjugated eye deviation to the right, and dysarthria 6 hours after procedure. However, the patient had full recovery after optimal medical treatment, including a bolus of heparin, continuous use of vasopressor, and hemodilution with fluid replacement. This patient showed sufficient platelet aggregation inhibitory effects (i.e., ADP 50\%: collagen 26\% in LTA and PRU 184: ARU 408 in VerifyNow) 3 days before surgery ( 5 days after the start of oral administration). On the other hand, the patient who had subcutaneous hematoma showed increase of platelet aggregation inhibitory effects (i.e., ADP/collagen in LTA was preoperative; $53 \% / 46 \%$, postoperative; $48 \% / 52 \%$, post-discharge; $36 \% / 32 \%$, respectively). According to the result, we reduced the dose of clopidogrel from 75 to $50 \mathrm{mg}$ /day. Thus, the subcutaneous hematoma was improved and the patient had no other complications after the dose adjustment.

All 30 patients underwent postoperative magnetic resonance imaging (MRI). The MRIs revealed that 23 of the patients had a new infarct lesion, but 22 of them were asymptomatic except for the patient with stent thrombosis.

\section{Discussion}

We investigated the efficacy of platelet aggregation monitoring and the changes of platelet aggregation inhibitory effect during continuation of dual antiplatelet therapy for stent-assisted coil embolization of cerebral aneurysm. The results revealed that the monitoring improved patient safety, and continuation of dual antiplatelet therapy caused increase in platelet aggregation inhibitory effect.
Since clopidogrel resistance is considered a contributing factor of thromboembolic complications, the use of strict criteria for determining clopidogrel efficacy is expected. When gauging platelet inhibition responsiveness with the VerifyNow P2Y12 assay and the associated impact on thrombosis and safety (GRAVITAS trial) in the field of cardiovascular medicine, the number of cardiovascular events occurring within the first 60 postoperative days significantly decreased when PRU $\leq 208$ was used as a cutoff value, although there was no significant difference when PRU $\leq 230$ was used. ${ }^{17,19)}$ Because there is currently no definite consensus regarding multiple antiplatelet therapy during intracranial stent placement, ${ }^{9,10,17,19)}$ indicators with established evidence in cardiovascular medicine are often used as PRU cut-off values. However, applying evidence from cardiovascular medicine to cerebral endovascular treatment is not always appropriate because intracranial arteries are more likely to show clearer symptoms than coronary arteries, and these vascular systems can differ in terms of vascular diameters, perfusion pressures, platelet aggregation induction, etc. ${ }^{3,11,12)}$ Therefore, although the development of criteria for cerebral endovascular treatment is urgently needed, a large-scale prospective study may be the most effective way to achieve such a guideline. However, insufficient inhibition of platelet aggregation may lead to very severe thromboembolic complications, making the conduction of such a study in the routine clinical setting very difficult.

On the other hand, we demonstrated that the inhibitory effects of clopidogrel on ADP-induced platelet aggregation and those of aspirin on collagen-induced platelet aggregation increased over time. In a previous report, Delgado et al. ${ }^{7)}$ defined the patients with a cutoff value of PRU $\leq 60$ in VerifyNow as clopidogrel hyper-responders. However, a similar definition of clopidogrel hyper-responder in LTA could not be found. Moreover, there were few reports about the increase in the inhibitory effects of aspirin. In this study, we experienced only one hemorrhagic complication as 
subcutaneous hematoma associated with these effects. Therefore, we could not evaluate the correlation between these effects and hemorrhagic complication, and whether each patient was a clopidogrel hyper-responder or not. According to Sibbing et al., ${ }^{20)}$ the risk of major bleeding is 3.5 times higher in clopidogrel hyper-responders than that in patients at risk for hemorrhagic complications during cardiovascular procedures. In addition, a meta-analysis by Li et al. ${ }^{21)}$ demonstrated that the risk of hemorrhagic complications is significantly higher in these populations. Likewise, in the field of cerebral endovascular treatment, Delgado et al. ${ }^{7)}$ and Goh et al. ${ }^{22)}$ reported a significant increase in hemorrhagic complications in patients who were hyper-responsive to clopidogrel. In addition, Pandya et al. ${ }^{18)}$ reported that the inhibitory effect of clopidogrel on ADP-induced platelet aggregation increases depending on therapy duration rather than on dose. In a study of Delgado Almandoz et al., ${ }^{23)} 59 \%$ of PRU normo-responders were later converted to hyper-responders. Furthermore, Endo et al. ${ }^{24)}$ showed that continuation of dual-antiplatelet therapy consisting of aspirin and clopidogrel induced delayed clopidogrel hyper-response and was associated with postoperative hemorrhagic complications. Therefore, excessive inhibition should be avoided to prevent hemorrhagic complications.

The preoperative use of platelet aggregation monitoring will likely be emphasized as a means to avoid perioperative thromboembolic complications. However, the results of this study suggest that postoperative platelet aggregation monitoring is also useful for assessing the risk of excessive platelet aggregation inhibition (due to continuous, postoperative administration) and hemorrhagic complications. Therefore, continuous monitoring of platelet aggregation may be important during the continuous administration of postoperative antiplatelet agents. Taking these results into consideration, we now tailor a regimen of platelet inhibition according to the result of LTA. Specifically, we administer aspirin (100 mg/day) and clopidogrel (75 mg/day) to all patients at least 10 days before surgery. If necessary, we reduce the dosage of each drug (clopidogrel; $50 \mathrm{mg} /$ day, $25 \mathrm{mg}$ /day, or $12.5 \mathrm{mg}$ /day and aspirin; $81 \mathrm{mg} /$ day) according to postoperative LTA results and hemorrhagic complications (i.e., subcutaneous hemorrhage, nasal bleeding, etc.). LTA is repeated after the drug adjustment.

This study has some limitations. First, the power of the study may be insufficient to have confidence in the results because our study was a single-center retrospective study with a small sample size. We searched for an optimal antiplatelet regimen by trying various regimens composed of aspirin (100 mg/day), clopidogrel (75 mg/day, $50 \mathrm{mg} /$ day and $25 \mathrm{mg} /$ day), and cilostazol (50 mg/day and $100 \mathrm{mg} /$ day) during the early days of stent-assisted coil embolization, and we reduced preoperative dose of clopidogrel to $50 \mathrm{mg} /$ day according to body weight, especially for female patients. In addition, we had a small sample size with preoperative VerifyNow measurement during the late study period because we had considered the results of LTA to be fairly reliable and the running cost of VerifyNow was very high. Second, we could not define the threshold for clopidogrel hyper-responder in LTA, and an association between the postoperative excessive inhibition of platelet aggregation and hemorrhagic complications. Third, we could not detect when the gradual increase in the inhibition effect of platelet aggregation has reached its plateau. Finally, the data may only represent individuals of one ethnic group; therefore, the influence of ethnicity on the results is unknown.

\section{Conclusion}

In this study, we examined the benefits of monitoring multiple antiplatelet therapies during stent-assisted embolization of intracranial aneurysms. We demonstrated the importance of continuous monitoring, postoperatively, to detect excessive inhibition of platelet aggregation. Future randomized studies are needed to clarify the efficacy and pharmacology of multiple antiplatelet therapy for intracranial stent placement.

\section{Acknowledgment}

The authors would like to acknowledge Yumiko MitomeMishima, Department of Neurosurgery, Juntendo University School of Medicine for her contribution to the additional data collection for this study, and Mikio Nakajima, Department of Clinical Epidemiology and Health Economics, The University of Tokyo for his contribution to the statistical analysis for this study.

\section{Disclosure Statement}

All authors have no conflict of interest to declare.

\section{References}

1) Yamada NK, Cross DT, Pilgram TK, et al: Effect of antiplatelet therapy on thromboembolic complications of elective coil embolization of cerebral aneurysms. AJNR Am J Neuroradiol 2007; 28: 1778-1782. 
2) Kang HS, Han MH, Kwon BJ, et al: Is clopidogrel premedication useful to reduce thromboembolic events during coil embolization for unruptured intracranial aneurysms? $\mathrm{Neu}$ rosurgery 2010; 67: 1371-1376; discussion 1376.

3) Riedel CH, Tietke M, Alfke K, et al: Subacute stent thrombosis in intracranial stenting. Stroke 2009; 40: 1310-1314.

4) Piotin M, Blanc R, Spelle L, et al: Stent-assisted coiling of intracranial aneurysms: clinical and angiographic results in 216 consecutive aneurysms. Stroke 2010; 41: 110-115.

5) Nishido H, Piotin M, Bartolini B, et al: Analysis of complications and recurrences of aneurysm coiling with special emphasis on the stent-assisted technique. AJNR Am J Neuroradiol 2014; 35: 339-344.

6) Oran I, Cinar C, Bozkaya H, et al: Tailoring platelet inhibition according to multiple electrode aggregometry decreases the rate of thrombotic complications after intracranial flow-diverting stent implantation. J Neurointerv Surg 2015; 7: 357-362.

7) Delgado Almandoz JE, Crandall BM, Scholz JM, et al: Pre-procedure P2Y12 reaction units value predicts perioperative thromboembolic and hemorrhagic complications in patients with cerebral aneurysms treated with pipeline embolization device. J Neurointerv Surg 2013; 5: iii3-iii10.

8) Rossen JD, Chalouhi N, Wassef SN, et al: Incidence of cerebral ischemic events after discontinuation of clopidogrel in patients with intracranial aneurysms treated with stent-assisted techniques. J Neurosurg 2012; 117: 929-933.

9) Bonello L, Tantry US, Marcucci R, et al: Consensus and future directions on the definition of high on-treatment platelet reactivity to adenosine diphosphate. J Am Coll Cardiol 2010; 56: 919-933.

10) Stone GW, Witzenbichler B, Weisz G, et al: Platelet reactivity and clinical outcomes after coronary artery implantation of drug-eluting stents (ADAPT-DES): a prospective multicentre registry study. Lancet 2013; 382: 614-623.

11) Leslie-Mazwi TM: Antiplatelet testing in neurointervention: we cannot ignore the signs. J Neurointerv Surg 2013; 5: 277-279.

12) Chandra RV: Point of care platelet function testing in routine neurointerventional care is unjustified. $J$ Neurointerv Surg 2013; 5: 280-282.

13) Hussein HM, Emiru T, Georgiadis AL, et al: Assessment of platelet inhibition by point-of-care testing in neuroendovascular procedures. AJNR Am J Neuroradiol 2013; 34: 700-706.

14) von Beckerath N, Pogatsa-Murray G, Wieczorek A, et al: Correlation of a new point-of-care test with conventional optical aggregometry for the assessment of clopidogrel responsiveness. Thromb Haemost 2006; 95: 910-911.

15) Sibbing D, Braun S, Jawansky S, et al: Assessment of ADP-induced platelet aggregation with light transmission aggregometry and multiple electrode platelet aggregometry before and after clopidogrel treatment. Thromb Haemost 2008; 99: 121-126.

16) Gremmel $T$, Steiner $S$, Seidinger $D$, et al: Comparison of methods to evaluate clopidogrel-mediated platelet inhibition after percutaneous intervention with stent implantation. Thromb Haemost 2009; 101: 333-339.

17) Price MJ, Berger PB, Teirstein PS, et al: Standard- vs highdose clopidogrel based on platelet function testing after percutaneous coronary intervention: the GRAVITAS randomized trial. JAMA 2011; 305: 1097-1105.

18) Pandya DJ, Fitzsimmons BF, Wolfe TJ, et al: Measurement of antiplatelet inhibition during neurointerventional procedures: the effect of antithrombotic duration and loading dose. J Neuroimaging 2010; 20: 64-69.

19) Price MJ, Angiolillo DJ, Teirstein PS, et al: Platelet reactivity and cardiovascular outcomes after percutaneous coronary intervention: a time-dependent analysis of the gauging responsiveness with a VerifyNow P2Y12 assay: impact on thrombosis and safety (GRAVITAS) trial. Circulation 2011; 124: 1132-1137.

20) Sibbing D, Schulz S, Braun S, et al: Antiplatelet effects of clopidogrel and bleeding in patients undergoing coronary stent placement. Thromb Haemost 2010; 8: 250-256.

21) Li Y, Tang HL, Hu YF, et al. The gain-of-function variant allele CYP2C19* 17: a double-edged sword between thrombosis and bleeding in clopidogrel-treated patients. Thromb Haemost 2012; 10: 199-206.

22) Goh C, Churilov L, Mitchell P, et al: Clopidogrel hyper-response and bleeding risk in neurointerventional procedures. AJNR Am J Neuroradiol 2013; 34: 721-726.

23) Delgado Almandoz JE, Kadkhodayan Y, Crandall BM, et al: Variability in initial response to standard clopidogrel therapy, delayed conversion to clopidogrel hyper-response, and associated thromboembolic and hemorrhagic complications in patients undergoing endovascular treatment of unruptured cerebral aneurysms. J Neurointerv Surg 2014; 6: 767-773.

24) Endo H, Kataoka T, Ogino $T$, et al: Delayed clopidogrel hyper-response after neuroendovascular therapy associated with hemorrhagic complications. JNET J Neurovasc Ther 2017; 11: 570-574. 